\title{
Outage Analysis in SWIPT Enabled Spectrum Sharing D2D Communication for loT Applications
}

This paper was downloaded from TechRxiv (https://www.techrxiv.org).

\section{LICENSE}

CC BY 4.0

SUBMISSION DATE / POSTED DATE

29-07-2021 / 02-08-2021

\section{CITATION}

Ghosh, Sutanu; Acharya, Tamaghna; Maity, Santi P. (2021): Outage Analysis in SWIPT Enabled Spectrum Sharing D2D Communication for loT Applications. TechRxiv. Preprint.

https://doi.org/10.36227/techrxiv.15073047.v1

$\mathrm{DOI}$

10.36227/techrxiv.15073047.v1 


\title{
Outage Analysis in SWIPT Enabled Spectrum Sharing D2D Communication for IoT Applications
}

\author{
Sutanu Ghosh, Student Member, IEEE, Tamaghna Acharya, Member, IEEE, Santi P. Maity, Member, IEEE
}

\begin{abstract}
This paper reports a relative performance analysis of decode-and-forward (DF) and amplify-and-forward (AF) relaying in a multi-antenna cooperative cognitive radio network (CCRN) that supports device-to-device (D2D) communications using spectrum sharing technique in cellular network. In this work, cellular system is considered as primary and internet of things devices (IoDs), engaged in D2D communications, are considered as secondary system. The devices access the licensed spectrum by means of the cooperation in two-way primary communications. Furthermore, IoDs are energized through the harvesting of energy from radio frequency (RF) signals, using simultaneous wireless information and power transfer (SWIPT) protocol. Closed form expressions of the outage probability for both cellular and D2D communications are derived and the impact of various design parameters for both $\mathrm{AF}$ and $\mathrm{DF}$ relaying techniques are studied. Based on the simulation results, it is found that the proposed spectrum sharing protocol, for both DF relaying and $A F$ relaying schemes, outperform a similar network architecture in terms of spectrum efficiency. It is also observed that the performance of the proposed system using DF relaying is $\sim \mathbf{1 6 8} \%$ better compared to AF relaying scheme in term of peak energy efficiency at same transmit power.
\end{abstract}

Index Terms-Simultaneous wireless information and power transfer, Spectrum sharing, D2D communication, cooperative cognitive radio network, outage analysis.

\section{INTRODUCTION}

Internet-of-things (IoT) is now under extensive development phase to support the needs of short packet (few information bytes) delivery (through frequency access or sharing) at ultrahigh reliability (low outage) and low latency (through efficient routing) for the specific applications like, smart health-care, smart transportation, smart grid, etc. Device-to-Device (D2D) communication looks promising in IoT networks due to enhanced battery life and service availability [1]. It also improves the proximity gain, pairing gain of radio spectrum without any involvement of cellular base station (BS) specifically when the radio frequency spectrum is shared by both D2D and cellular users [2]. Congestion due to proliferation of wireless devices in unlicensed band urges the essence of sharing of the spectrum, originally licensed to cellular users, so as to support future IoT communication [3].

Cooperative cognitive radio network $(\mathrm{CCRN})$ refers to a network model that facilitates overlay mode of cognitive radio

Sutanu Ghosh and Tamaghna Acharya are with the Department of Electronics and Telecommunication Engineering, Indian institute of Engineering Science and Technology, Shibpur, Howrah, West Bengal, 711103, India. (Email: sutanu99@gmail.com, tamaghna.acharya@ieee.org).

Santi P. Maity is with the Department of Information Technology, Indian institute of Engineering Science and Technology, Shibpur, Howrah, West Bengal, 711103, India. (E-mail: santipmaity@it.iiests.ac.in). enabled spectrum sharing [4] and could be applied in D2D communication in $5 \mathrm{G}$ heterogeneous networks (HetNets). Following this, the pair of IoT devices (IoDs), using D2D communication as unlicensed users, may be viewed as secondary users (SUs) while cellular nodes (i.e. evolved Node-B (eNB) and user equipment (UE)) can be modelled as primary users (PUs). IoDs can access the licensed spectrum of cellular system while agreeing to relay the signals of the latter, thereby improving the reliability of their communication over the fading wireless channel. In literature, two-way communications [5] based on CCRN is studied to achieve higher spectrum efficiency (SE) over the one-way relaying [6]. The outage performance of the two-way CCRN is studied in [5] and the impact of different relaying schemes on spectrum efficient network operation is reported.

Recently, radio frequency energy harvesting (RF-EH) based relaying using simultaneous wireless information and power transfer (SWIPT) protocol has been under extensive investigation to enhance network lifetime and reliability of wireless communication [7]-[9]. SU transmitter of CCRN may follow amplify-and-forward (AF) or, decode-and-forward (DF) relaying to support PU communication and send its own message to SU receiver using the energy harvested from the received PU signal. Relay node harvests the energy from the PU signal by following power splitting (PS) or, time switching (TS) based SWIPT protocols [10]. Performance of unidirectional or bidirectional communications using AF and DF relay aided SWIPT networks are analysed in [11]-[14]. The system outage performances of one-way DF relay assisted communication in CCRN is studied in [11] over a Nakagami fading channel. In [12], PU and SU outage performances are studied in two-way CCRN using AF relay assisted network. The authors of [12] also show the impact of energy conversion efficiency on the system energy efficiency (EE). The performance of similar network model is studied in [13] using DF relay. Based on the study of [13], it is shown that DF relay-assisted twoway communications are significantly more spectrum efficient than one-way communication [11] using PS relaying (PSR) protocol. However, privacy and security issues may be of concern as DF relay is required to decode PU message during the relaying process [15]. On the contrary, in AF relaying, PU signal needs not be decoded at the relaying node, although, it is often considered to be an energy inefficient approach as noise gets amplified by the relay node. The performance improvement of SU communication over [13] is studied in [14] using bidirectional SU communication. Needless to mention that the presence of multiple antennas at the source and/or at the relay nodes in any SWIPT enabled relay assisted commu- 
nication over fading channel, not only improves the reliability of information transfer but also enhances $\mathrm{EH}$ at the latter. Motivated by this, a preliminary study on the performance of the same system model [13] with multiple antenna PUs is presented in [16]. In this paper, a comparative study between $\mathrm{DF}$ and AF relaying scheme is presented following PS based relaying protocol in CCRN.

\section{Scope and Contributions:}

In the literature the impact of multiple antenna and the performance comparison between SWIPT enabled DF and AF relay assisted communication are studied separately. The findings in these works [12], [13], [16] motivate us to study the impact of multiple antenna on SWIPT enabled two-phase two-way PU and one-way SU communication for both DF and AF relay assisted CCRN. In our work, SU transmitter acts as relay and harvests its energy required for relaying from RF signals of PU transmissions. In two-way relay assisted communications, if EH based on TSR protocol is followed, then total transmission frame needs splitting in at least three non-overlapping time slots : (i) one common slot for $\mathrm{EH}$ from the RF signals transmitted by the eNB $\left(\mathrm{PU}_{1}\right)$ and $\mathrm{UE}$ $\left(\mathrm{PU}_{2}\right)$ and (ii) one slot for information processing of the RF signal transmitted by $\mathrm{PU}_{1}$ and $\mathrm{PU}_{2}$; (iii) finally, one slot for simultaneous relaying to $\mathrm{PU}_{1}$ and $\mathrm{PU}_{2}$ by $\mathrm{SU}_{1}$. Since, in each frame, information transfer from PUs-to-SU and relaying from SU to PUs vary linearly with the time of transmission, $\mathrm{EH}$ and information processing are not done here in separate non-overlapping time slots aiming to improve the end-to-end rate of information transfer between PUs. Moreover, PSR protocol is reported to perform better in terms of SE over TSR protocol for a wide range of SNR [10]. As one of the goal of this work is to achieve high SE at relatively wide and higher SNR region, therefore, PSR protocol is used in this work as done in [13]. The work in [13] follows threephase DF relay assisted single-antenna communication in a CCRN framework. However, it does not explore the impact of multi-antenna on SE and EE aspects of the proposed D2D operation in $5 \mathrm{G}$ HetNets. Furthermore, two-phase communication is preferable to three-phase for enhancement in the system throughput. These lead us to explore the problem of spectrum sharing for bidirectional cellular communication and unidirectional D2D communication simultaneously using twophase PSR protocol. To offer improved SE, the present cellular system uses multiple antennas, to avail the benefits of spatial diversity and spatial multiplexing as trend seen on 5G [17]. In PSR protocol, although power splitting factor becomes a design variable, its value is easy to change/control in physical layer data transmission. Now, if non-equal times slots for $\mathrm{EH}$ and relaying are used, its optimal value calculation not only makes mathematical analysis complicated, but also this design variable ${ }^{1}$ will make the synchronization in data transmission mode more complex. Furthermore, IoD node is used as a relay in two-way communications between eNB and UE, and their RF signals in multiple access channel (MAC) phase seem to be sufficient to harvest the required energy for data transmission.

\footnotetext{
${ }^{1}$ as because the non-equal time slots includes another design variable
}

Henceforth, in this work, two-phase PSR protocol is used wherein time allocation for $\mathrm{EH}$ in MAC phase and relaying in broadcast (BC) phase are made equal following the case of ideal receiver [10].

To make this study more general, non-equal number of antennas are considered at two end of PU system. The nodes engaged in D2D communication are assumed to be equipped with necessary hardware to harvest energy from the RF signals of the cellular users. EH meets the energy requirement of the transmitting node for D2D communication to relay (AF/DF) the signals of the cellular users and also its own message transmission over the cellular spectrum simultaneously. The main objective of this work is to highlight the relative improvements in SE and EE performance of spectrum sharing for the twoway $\mathrm{AF} / \mathrm{DF}$ relaying system, as the primary message sources evolve from a single antenna to a multi-antenna system. The contributions of this study can be summarized as follows.

- A novel CCRN architecture with multi-antenna PU system is proposed where two-phase protocol supports two-way SWIPT enabled communications between the pair of cellular users (PUs) and also one-way D2D (SU-to-SU) communication. Two fold benefits, the first one is the improved SE due to multi-antenna and the other one is the throughput improvement due to two-phase protocol are achieved.

- Closed form expressions of both PU and SU outage probability are derived for both $\mathrm{DF}$ and $\mathrm{AF}$ relay assisted communication using PSR protocol for multi-antenna CCRN framework. Simulation results closely match the analytical expressions.

- The exact dependence of the system performance on various parameters like power sharing factor, power splitting factor, transmission power are also shown through the simulation results. DF relaying mostly performs better than AF relaying. As we have used two-phase spectrum sharing protocol, therefore both the relaying mechanisms show almost equal spectrum efficiency over the similar existing network architecture [13]. However, AF relaying is found to be better in terms of an incremental improvement in SE with the increase in the number of antennas.

The various symbols used are introduced in Table- 1 . The remaining part of the paper is arranged as follows. The system model is described in Section II and communication protocol description is given in Section III. The outage performance of both PU and SU are analysed in Section IV. The necessary simulation and the numerical results in terms of the various system parameters are presented in Section V. Finally, the paper is concluded in Section VI.

\section{SYSTEM MODEL}

\section{A. Assumptions and Notations}

The system model consists of a pair of eNB and UE, located at the edge of the femto-cell [18] in long term evolution (LTE) network architecture as depicted in Fig. 1. The eNB and UE are equipped with multiple antennas $\mathrm{N}_{a}$ and $\mathrm{N}_{b}$, respectively. In absence of the direct communication link (due to some obstacles or poor channel conditions), the cellular nodes (eNB and UE) intend to exchange their information via a single 
TABLE I

SYMBOLS AND DEFINITIONS

\begin{tabular}{l|l}
\hline \hline Symbols & Definitions \\
\hline \hline$X_{s}$ & Signal transmission between $\mathrm{IoD}_{1}\left(\mathrm{SU}_{1}\right)$ and $\mathrm{IoD}_{2}\left(\mathrm{SU}_{2}\right)$ \\
\hline$P_{p_{1}}, P_{p_{2}}$ & Transmission power of $\mathrm{PU}_{1}, \mathrm{PU}_{2}$ \\
\hline $\mathbf{h}_{i}$ & Channel gain of link $\mathrm{PU}_{i} \rightarrow \mathrm{SU}_{1}(i \in 1,2)$ \\
\hline $\mathbf{g}_{i}$ & Channel gain of link $\mathrm{PU}_{i} \rightarrow \mathrm{SU}_{2}(i \in 1,2)$ \\
\hline$D_{i}$ & Distance between $\mathrm{PU}_{i}$ and $\mathrm{SU}_{1}(i \in 1,2)$ \\
\hline$D_{j}$ & $\begin{array}{l}\text { Distance between } \mathrm{PU}_{i} \text { and } \mathrm{SU}_{2}(\text { for } i=1, j=4 \text { and } \\
i=2, j=5)\end{array}$ \\
\hline$n_{s u}, n_{p u}$ & Received noise at $\mathrm{SU}, \mathrm{PU}_{1}$ respectively \\
\hline$h_{3}$ & Channel gain of link $\mathrm{SU}_{1} \rightarrow \mathrm{SU}_{2}$ \\
\hline$\rho$ & Power splitting factor of $\mathrm{SU}_{1}$ \\
\hline$\alpha$ & Power sharing factor at $\mathrm{SU}_{1}$ \\
\hline $\mathrm{L}$ & Distance between $\mathrm{PU}_{1}$ and $\mathrm{PU}_{2}$ \\
\hline$D_{3}$ & Distance between $\mathrm{SU}_{1}$ and $\mathrm{SU}_{2}$ \\
\hline$m$ & Path loss exponent \\
\hline$R_{P U}, R_{S U}$ & Energy conversion efficiency at $\mathrm{SU}_{1}$ \\
\hline
\end{tabular}

antenna IoT device (IoD) (denoted as $\mathrm{IoD}_{1}$ in Fig. 1.), aiming to achieve the PU target rate at each side. Simultaneously, $\mathrm{IoD}_{1}$ sends its own message signal to $\mathrm{IoD}_{2}$ to meet a target rate of $R_{S U}$. Both of them use single antenna for communication. Here $\mathrm{eNB}$ and $\mathrm{UE}$ are considered as $\mathrm{PU}_{1}$ and $\mathrm{PU}_{2}$, respectively and $\mathrm{IoD}_{1}$ and $\mathrm{IoD}_{2}$ are modelled as $\mathrm{SU}_{1}$ and $\mathrm{SU}_{2}$, respectively.

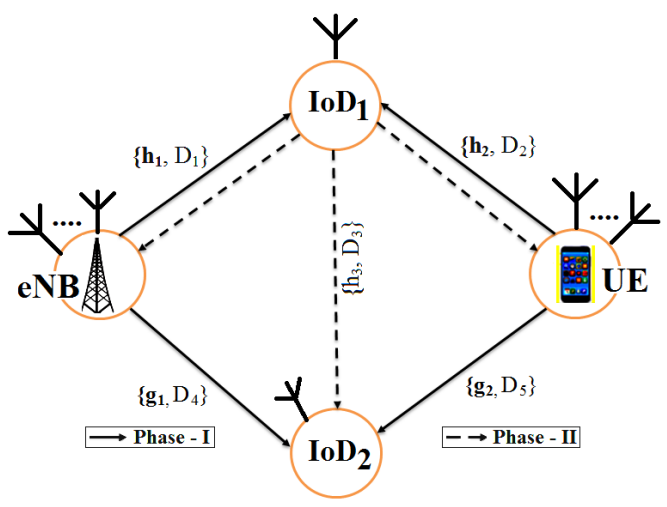

Fig. 1. System model

We assume that $\mathrm{PU}_{i},(i \in 1,2)$ uses fixed power supply, i.e., $P_{p_{i}}$ while $\mathrm{SU}_{1}$ is powered through harvested energy from PUs' RF signals, using SWIPT, for relaying PUs' messages and data transmission to $\mathrm{SU}_{2}$. We consider both $\mathrm{DF}$ and $\mathrm{AF}$ relaying mechanisms to support the two-way PU communications. Here $\mathbf{h}_{i}=\left[h_{i, 1}, h_{i, 2}, \ldots, h_{i, N_{p}}\right],\left(\mathbf{N}_{p}\right.$ $\left.\in \mathbf{N}_{a}, \mathbf{N}_{b}\right)$ and $\mathbf{g}_{i}=\left[g_{i, 1}, g_{i, 2}, \ldots, g_{i, N_{p}}\right]$, (i $\left.\in 1,2\right)$ are the vector channel coefficients from the multiple-antenna $\mathrm{PU}_{i}$ to $\mathrm{SU}_{1}$ and $\mathrm{PU}_{i}$ to $\mathrm{SU}_{2}$, respectively, where $h_{i, n} \sim \mathcal{C N}(0,1)$ and $g_{i, n} \sim \mathcal{C N}(0,1)$. Channel between $\mathrm{SU}_{1} \rightarrow \mathrm{SU}_{2}$ is represented by $h_{3}$, where $h_{3} \sim \mathcal{C N}\left(m_{k}, 1\right) . \mathrm{m}_{k}$ is Nakagami shaping parameter. Since, there is no direct link between PU nodes, therefore, independent and identically distributed (i.i.d) Rayleigh fading is considered for all the links between PU and SU nodes. However, Nakagami-m fading is considered for the direct link between $\mathrm{SU}_{1}$ and $\mathrm{SU}_{2}$, since they communicate in D2D mode. If Channel state information (CSI) is available at PU transmitter then efficient power allocation leads to achieve higher data rate. However, this adds to the system complexity in terms of the requirement of time/bandwidth for the feedback link. Hence, the requirement of instantaneous CSI at $\mathrm{PU}_{i}(i=1,2)$ is not considered in the current study. Twoantenna transmission technique to achieve full diversity order in absence of instantaneous transmit CSI, is already illustrated using Alamouti coding [19] technique. Its extension for multiantenna system is proposed in full-diversity space-time code (like, generalized ABBA (GABBA) codes [20]). The GABBA codes are also useful option over orthogonal space-time block codes (OSTBC) and quasi orthogonal space-time block codes (QOSTBC) to overcome the drawback of full diversity gain with full code rate [20]. Therefore, GABBA codes are used at the PU nodes in the given system to achieve higher diversity gain with full code rate. Since SUs are allowed to use CRoverlay mode for PU spectrum access, it is assumed that they have the necessary codebook for decoding of PUs' information bearing signals. Normally, in space-time code, power is uniformly distributed among the transmitting antennas. The distances between the users $\mathrm{PU}_{1}-\mathrm{SU}_{1}, \mathrm{PU}_{2}-\mathrm{SU}_{1}, \mathrm{PU}_{1}-\mathrm{SU}_{2}$, $\mathrm{PU}_{2}-\mathrm{SU}_{2}$ and $\mathrm{SU}_{1}-\mathrm{SU}_{2}$ are given by $D_{1}, D_{2}, D_{4}, D_{5}$ and $D_{3}$, respectively with ' $m$ ' as the path-loss exponent. Here $n_{s u}$ and $n_{p u}$ indicate the received noise at SU and PU, respectively. The noise is assumed to be additive white Gaussian noise (AWGN) with zero (0) mean and the variance $\sigma^{2}$.

\section{B. Protocol description}

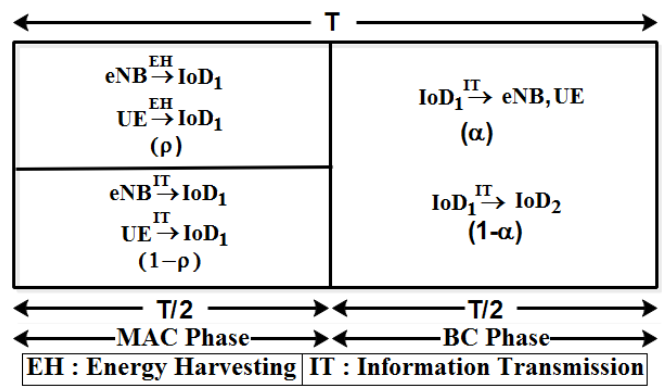

Fig. 2. Transmission frame structure in the two-way PSR protocol

The two-way communications between $\mathrm{PU}_{1}$ and $\mathrm{PU}_{2}$ via $\mathrm{SU}_{1}$ take place in two time phases viz., MAC and $\mathrm{BC}$ phase, as shown in Fig. 2. Total time frame duration $T$ is equally divided for MAC phase and $\mathrm{BC}$ phase. In the MAC phase, both $\mathrm{PU}_{1}$ and $\mathrm{PU}_{2}$ simultaneously transmit their information signals to $\mathrm{SU}_{1} . \mathrm{SU}_{1}$ is able to harvest energy from part of the received signal using PSR protocol. In the case of AF relaying, $\mathrm{SU}_{1}$ broadcasts an amplified $\mathrm{PU}$ signals superimposed with its own signal $X_{s}$ in the BC phase, using the total harvested energy. The PU receivers seem to receive the signals using the maximal-ratio-combining (MRC) and separate the desired signal using the self-interference cancellation (SIC) [13]. Now, decoding of $\mathrm{SU}_{1}$ 's message at $\mathrm{SU}_{2}$ is performed in the two phases; first the stronger PU signal is decoded considering $\mathrm{SU}_{1}$ 's desired signal as interference. Once the strong PU signal is separated from the received signal in the the first phase, $\mathrm{SU}_{2}$ decodes the desired $\mathrm{SU}_{1}$ 's signal in presence of channel noise. 
In the case of $\mathrm{DF}$ relaying, $\mathrm{SU}_{1}$ first decodes the PUs' message signals received in the MAC phase, and then broadcasts a network coded primary signal superimposed on the secondary signal $X_{s}$ in the BC phase. Both the PU nodes decode the network coded (XOR operation) PU signal in presence of SU interfering signal and noise, while $\mathrm{SU}_{2}$ decodes $\mathrm{SU}_{1}$ signal by cancelling PU signal's based on the PUs' messages received previously in the MAC Phase.

\section{RATE AND OUTAGE ANALYSIS IN DF RELAYING}

\section{A. Rate Analysis}

Now the received power in MAC phase at $S U_{1}$ from $\mathrm{PU}_{1}$ and $\mathrm{PU}_{2}$ can be expressed as follow $\mathrm{s}^{2}$ [21].

$$
P_{S U_{1}}^{(1)}=\frac{P_{p_{1}} \sum_{n=1}^{N_{a}\left|h_{1, n}\right|^{2}}}{N_{a}\left(D_{1}\right)^{m}}+\frac{P_{p_{2}} \sum_{p=1}^{N_{b}}\left|h_{2, p}\right|^{2}}{N_{b}\left(D_{2}\right)^{m}} .
$$

where $P_{p_{1}}$ and $P_{p_{2}}$ are the transmit powers of $\mathrm{PU}_{1}$ and $\mathrm{PU}_{2}$, respectively.

Similarly, the received power at $S U_{2}$ from $\mathrm{PU}_{1}$ and $\mathrm{PU}_{2}$ can be expressed as

$$
P_{S U_{2}}^{(1)}=\frac{P_{p_{1}} \sum_{n=1}^{N_{a}}\left|g_{1, n}\right|^{2}}{N_{a}\left(D_{4}\right)^{m}}+\frac{P_{p_{2}} \sum_{p=1}^{N_{b}}\left|g_{2, p}\right|^{2}}{N_{b}\left(D_{5}\right)^{m}} .
$$

A part $\rho$ (referred to as power splitting factor in the subsequent discussion) of the received signal power at $\mathrm{SU}_{1}$ is used for $\mathrm{EH}$, and the rest $(1-\rho)$ portion is used for information processing. The harvested energy in MAC phase is given by

$$
E_{s}=\frac{\eta \rho T}{2}\left(\frac{P_{p_{1}} \sum_{n=1}^{N_{a}}\left|h_{1, n}\right|^{2}}{N_{a}\left(D_{1}\right)^{m}}+\frac{P_{p_{2}} \sum_{p=1}^{N_{b}}\left|h_{2, p}\right|^{2}}{N_{b}\left(D_{2}\right)^{m}}\right) .
$$

where $0<\eta<1$ represents the energy conversion efficiency. After power splitting, the received power in MAC phase at the receiver of $\mathrm{SU}_{1}$ for information processing is given by

$$
P_{S U_{1}}^{(1, I T)}=(1-\rho)\left[\frac{P_{p_{1}} \sum_{n=1}^{N_{a}\left|h_{1, n}\right|^{2}}}{N_{a}\left(D_{1}\right)^{m}}+\frac{P_{p_{2}} \sum_{p=1}^{N_{b}}\left|h_{2, p}\right|^{2}}{N_{b}\left(D_{2}\right)^{m}}\right] .
$$

Now following the linear EH model, the available power at $\mathrm{SU}_{1}$ for transmission in the $\mathrm{BC}$ phase is given by

$$
P_{s}=\eta \rho\left(\frac{P_{p_{1}} \sum_{n=1}^{N_{a}}\left|h_{1, n}\right|^{2}}{N_{a}\left(D_{1}\right)^{m}}+\frac{P_{p_{2}} \sum_{p=1}^{N_{b}}\left|h_{2, p}\right|^{2}}{N_{b}\left(D_{2}\right)^{m}}\right) .
$$

In the MAC phase, the successful decoding of the received signals from $\mathrm{PU}_{1}$ and $\mathrm{PU}_{2}$ is possible at $\mathrm{SU}_{1}$, if $R_{S U_{1}}^{(11)} \geq$ $R_{P U_{1}}, R_{S U_{1}}^{(12)} \geq R_{P U_{2}}$ and $R_{S U_{1}}^{\sum_{P}} \geq R_{P U_{1}}+R_{P U_{2}} ;\left(R_{P U_{1}} \geq\right.$ $R_{P U_{2}}$ ) [22], where $R_{P U_{1}}$ and $R_{P U_{2}}$ are the target rates of $\mathrm{PU}_{1}$ and $\mathrm{PU}_{2}$, respectively. Now,

$$
Q_{1}=\left\{\begin{array}{c}
R_{S U_{1}}^{(11)}=\frac{T}{2 T} \log _{2}\left(1+\frac{(1-\rho) P_{p_{1}} \sum_{n=1}^{N_{a}}\left|h_{1, n}\right|^{2}}{N_{a}\left(D_{1}\right)^{m} \sigma^{2}}\right), \\
R_{S U_{1}}^{(12)}=\frac{T}{2 T} \log _{2}\left(1+\frac{(1-\rho) P_{p_{2}} \sum_{p=1}^{N_{b}}\left|h_{2, p}\right|^{2}}{N_{b}\left(D_{2}\right)^{m} \sigma^{2}}\right), \\
R_{S U_{1}}^{\sum_{S T}}=\frac{T}{2 T} \log _{2}\left(1+\frac{(1-\rho) P_{p_{1}} \sum_{n=1}^{N_{a}}\left|h_{1, n}\right|^{2}}{N_{a}\left(D_{1}\right)^{m} \sigma^{2}}\right. \\
\left.+\frac{(1-\rho) P_{p_{2}} \sum_{p=1}^{N_{b}}\left|h_{2, p}\right|^{2}}{N_{b}\left(D_{2}\right)^{m} \sigma^{2}}\right) .
\end{array}\right.
$$

\footnotetext{
${ }^{2}$ In absence of CSI, $\mathrm{PU}_{1}$ and $\mathrm{PU}_{2}$ are assumed to use suitable space-time coding techniques, the details of which are not within the scope of our current study.
}

Similarly, in the MAC phase, the successful decoding of the received signals from $\mathrm{PU}_{1}$ and $\mathrm{PU}_{2}$ is possible at $\mathrm{SU}_{2}$, if $R_{S U_{2}}^{(11)} \geq R_{P U_{1}}, R_{S U_{2}}^{(12)} \geq R_{P U_{2}}$ and $R_{S U_{2}}^{\sum_{2}} \geq R_{P U_{1}}+R_{P U_{2}}$, where

$$
Q_{2}=\left\{\begin{array}{l}
R_{S U_{2}}^{(11)}=\frac{1}{2} \log _{2}\left(1+\frac{P_{p_{1}} \sum_{n=1}^{N_{a}}\left|g_{1, n}\right|^{2}}{N_{a}\left(D_{4}\right)^{m} \sigma^{2}}\right), \\
R_{S U_{2}}^{(12)}=\frac{1}{2} \log _{2}\left(1+\frac{P_{p_{2}} \sum_{p=1}^{N_{b}\left|g_{2, p}\right|^{2}}}{N_{b}\left(D_{5}\right)^{m} \sigma^{2}}\right), \\
R_{S U_{2}}^{\sum_{U_{2}}}=\frac{1}{2} \log _{2}\left(1+\frac{P_{p_{1}} \sum_{n=1}^{N_{a}}\left|g_{1, n}\right|^{2}}{N_{a}\left(D_{4}\right)^{m} \sigma^{2}}+\frac{P_{p_{2}} \sum_{p=1}^{N_{b}}\left|g_{2, p}\right|^{2}}{N_{b}\left(D_{5}\right)^{m} \sigma^{2}}\right) .
\end{array}\right.
$$

In DF relaying, $\mathrm{SU}_{1}$ uses $\alpha(0<\alpha<1)$ fraction of its total transmit power $P_{s}$ to relay the network-coded PU information and rest $(1-\alpha)$ fraction of $P_{s}$ is used to send its own independent message $X_{s}$ to $\mathrm{SU}_{2}$.

After MRC and cancellation of self-interference terms by applying SIC technique, the received signal-to-interferencenoise ratio (SINR) at $\mathrm{PU}_{i}\left(\mathrm{i} \in 1,2 ; \mathrm{N}_{p} \in \mathrm{N}_{a}, \mathrm{~N}_{b}\right)$ receivers in $\mathrm{BC}$ phase can be expressed as

$$
\gamma_{i}^{D F}=\frac{\frac{\alpha P_{s}}{\left(D_{i}\right)^{m}} \sum_{w=1}^{N_{p}}\left|h_{i, w}\right|^{2}}{\frac{(1-\alpha) P_{s}}{\left(D_{i}\right)^{m}} \sum_{w=1}^{N_{p}}\left|h_{i, w}\right|^{2}+\sigma^{2}}
$$

It is assumed that $\mathrm{SU}_{2}$, like $\mathrm{SU}_{1}$, succeeds in decoding the PUs' signals received in MAC phase. Based on these prior knowledges, $\mathrm{SU}_{2}$ is able to separate the desired $\mathrm{SU}$ signal from the PUs' interference received in $\mathrm{BC}$ phase [13]. Therefore, the received signal at $\mathrm{SU}_{2}$ can be rewritten as

$$
Y_{S U_{2}}^{(2, D F)}=\underbrace{\sqrt{\frac{(1-\alpha) P_{s}}{D_{3}^{m}}} h_{3} X_{s}}_{\text {Required signal }}+\underbrace{n_{s u}}_{\text {Noise }} .
$$

Now, the achievable rate at $\mathrm{PU}_{i}$ is given by (based on the $\mathrm{SINR}$ at $\left.\mathrm{PU}_{i}\right)\left(\mathrm{N}_{p} \in \mathrm{N}_{a}, \mathrm{~N}_{b}\right)$

$$
\begin{aligned}
& R_{P U_{i}}^{(2, D F)}=\frac{1}{2} \log _{2}\left\{1+\gamma_{i}^{D F}\right\} \\
= & \frac{1}{2} \log _{2}\left\{1+\frac{a_{i}^{\prime}\left(\sum_{n=1}^{N_{a}} a_{1}\left|h_{1, n}\right|^{2}+\sum_{p=1}^{N_{b}} b_{1}\left|h_{2, p}\right|^{2}\right) \sum_{w=1}^{N_{p}}\left|h_{i, w}\right|^{2}}{b_{i}^{\prime}\left(\sum_{n=1}^{N_{a}} a_{1}\left|h_{1, n}\right|^{2}+\sum_{p=1}^{N_{b}} b_{1}\left|h_{2, p}\right|^{2}\right) \sum_{w=1}^{N_{p}}\left|h_{i, w}\right|^{2}+1}\right\} .
\end{aligned}
$$

where $\quad a_{1}=\frac{\eta \rho P_{p_{1}}}{N_{a}\left(D_{1}\right)^{m} \sigma^{2}}, \quad b_{1}=\frac{\eta \rho P_{p_{2}}}{N_{b}\left(D_{2}\right)^{m} \sigma^{2}}, \quad a_{i}^{\prime}=\frac{\alpha}{\left(D_{i}\right)^{m}}$, $b_{i}^{\prime}=\frac{(1-\alpha)}{\left(D_{i}\right)^{m}}$.

Based on (9), the achievable rate at $\mathrm{SU}_{2}$ is expressed as

$$
\begin{aligned}
& R_{S U_{2}}^{(2, D F)}=\frac{1}{2} \log _{2}\left\{1+\frac{\frac{(1-\alpha) P_{s}}{D_{3}^{m}}\left|h_{3}\right|^{2}}{\sigma^{2}}\right\} \\
= & \frac{1}{2} \log _{2}\left\{1+c\left(\sum_{n=1}^{N_{a}} a_{1}\left|h_{1, n}\right|^{2}+\sum_{p=1}^{N_{b}} b_{1}\left|h_{2, p}\right|^{2}\right)\left|h_{3}\right|^{2}\right\} . \\
& \text { where } c=\frac{(1-\alpha)}{D_{3} m} .
\end{aligned}
$$

\section{B. Outage Probability Analysis}

An outage occurs when the achievable rate of data transmission on any transmission link falls below the target rate of data transmission. 
1) Outage Probability Analysis of Primary System: Based on the definition, PU outage probability ${ }^{3}$ using DF relaying mechanism can be determined as follows [13]:

$$
\begin{aligned}
& \mathscr{P}_{\text {out }}^{(P U, D F)}=1-\overbrace{[\underbrace{\mathscr{P}\left\{Q_{1}\right\}}_{\text {MAC phase }} \times \underbrace{\mathscr{P}\left\{\min \left(R_{P U_{1}}^{(2, D F)}, R_{P U_{2}}^{(2, D F)}\right) \geq R_{P U}\right\}}_{\text {BC phase }}]}^{\text {Success Probability }} \\
= & 1-\left[\mathscr{P}\left\{Q_{1}\right\} \times \mathscr{P}\left\{R_{P U_{1}}^{(2, D F)} \geq R_{P U}\right\} \times \mathscr{P}\left\{R_{P U_{2}}^{(2, D F)} \geq R_{P U}\right\}\right] .
\end{aligned}
$$

where $R_{P U}=\max \left\{R_{P U_{1}}, R_{P U_{2}}\right\}$

Applying (6), the success probability of data transmission between the $\mathrm{PU}$ nodes $\left(\mathrm{PU}_{1}\right.$ and $\left.\mathrm{PU}_{2}\right)$ and $\mathrm{SU}_{1}$ is expressed by following three conditions satisfied together.

$Q_{1}=\left\{\begin{array}{l}R_{S U_{1}}^{(11)}=\frac{1}{2} \log _{2}\left(1+A_{1} X_{1}\right) \geq R_{P U_{1}}, \\ R_{S U_{1}}^{(12)}=\frac{1}{2} \log _{2}\left(1+A_{2} Y_{1}\right) \geq R_{P U_{2}}, \\ R_{S U_{1}}^{\sum_{1}}=\frac{1}{2} \log _{2}\left(1+A_{1} X_{1}+A_{2} Y_{1}\right) \geq R_{P U_{1}}+R_{P U_{2}} .\end{array}\right.$

where $\quad A_{1}=\frac{(1-\rho) P_{p_{1}}}{N_{o}\left(D_{1}\right)^{m} \sigma^{2}}, \quad A_{2}=\frac{(1-\rho) P_{p_{2}}}{N_{b}\left(D_{2}\right)^{m} \sigma^{2}}$, $u_{1}=2^{\left(2 R_{P U_{1}}\right)}-1, u_{2}=2^{\left(2 R_{P U_{2}}\right)}-1$, $u_{3}=2^{\left(2 R_{P U_{1}}+2 R_{P U_{2}}\right)}-1$.

$X_{1}=\sum_{n=1}^{N_{a}}\left|h_{1, n}\right|^{2}$ and $Y_{1}=\sum_{p=1}^{N_{b}}\left|h_{2, p}\right|^{2}$ follow the same nature of gamma distribution with $N_{p}$ degree of freedom. The probability density function (PDF) can be expressed as $f_{X}(x)=\frac{x^{N_{p}-1} \exp \left(-\frac{x}{1 / N_{p}}\right)}{\Gamma\left(N_{p}\right)\left(1 / N_{p}\right)^{N_{p}}}$. Based on (13), $\mathscr{P}\left\{Q_{1}\right\}$ can be expressed as [23, Sec. 3.381.1, 3.381.3, 8.350.1, 8.352.1]

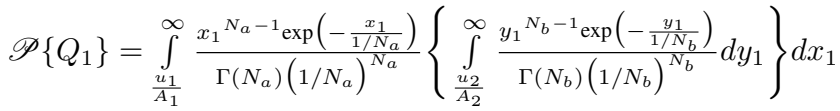

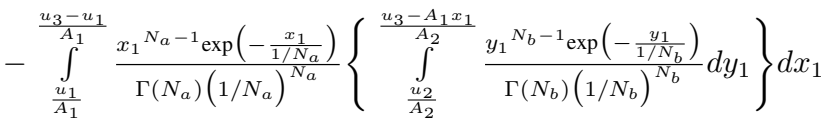

For simplification of mathematical analysis and better tractability,

the following numerical values are set, $N_{a}=\mathbf{2}$ and $N_{b}=1$

$$
\begin{aligned}
& =\left[\Gamma\left(2, \frac{2 u_{1}}{A_{1}}\right)-\left\{\gamma\left(2, \frac{2\left(u_{3}-u_{1}\right)}{A_{1}}\right)-\gamma\left(2, \frac{2 u_{1}}{A_{1}}\right)\right\}\right] \exp \left(-\frac{u_{2}}{A_{2}}\right) \\
& +\frac{4 \exp \left(-\frac{u_{3}}{A_{2}}\right)}{\left(2-\frac{A_{1}}{A_{2}}\right)^{2}}\left[\gamma\left\{2, \frac{\left(2-\frac{A_{1}}{A_{2}}\right)\left(u_{3}-u_{1}\right)}{A_{1}}\right\}-\gamma\left\{2, \frac{u_{1}\left(2-\frac{A_{1}}{A_{2}}\right)}{A_{1}}\right\}\right] .
\end{aligned}
$$

where $\Gamma(.,$.$) and \gamma(.,$.$) are the upper and the lower incomplete$ gamma function, respectively.

The probability of successful data transmission from $\mathrm{SU}_{1}$ to $\mathrm{PU}_{1}$ can be written as

$R_{P U_{1}}^{(2, D F)}=\frac{T}{2 T} \log _{2}\left\{1+\frac{a_{1}^{\prime}\left(\sum_{n=1}^{N a} a_{1}\left|h_{1, n}\right|^{2}+\sum_{p=1}^{N_{b}} b_{1}\left|h_{2, p}\right|^{2}\right) \sum_{n=1}^{N a}\left|h_{1, n}\right|^{2}}{b_{1}^{\prime}\left(\sum_{n=1}^{N a} a_{1}\left|h_{1, n}\right|^{2}+\sum_{p=1}^{N b} b_{1}\left|h_{2, p}\right|^{2}\right) \sum_{n=1}^{N a}\left|h_{1, n}\right|^{2}+1}\right\}$.

\footnotetext{
${ }^{3}$ Since $\mathrm{SU}_{1}$ is used as a relay to support $\mathrm{PU}$ communication, therefore, the successful information transmission from both the $\mathrm{PU}$ nodes to $\mathrm{SU}_{1}$ is essential in MAC phase for relaying the information in $\mathrm{BC}$ phase.
}

$$
\begin{aligned}
& \mathscr{P}\left\{R_{P U_{1}}^{(2, D F)} \geq R_{P U}\right\}=\mathscr{P}\left\{\frac{a_{1}^{\prime}\left(a_{1} X_{1}+b_{1} Y_{1}\right) X_{1}}{b_{1}^{\prime}\left(a_{1} X_{1}+b_{1} Y_{1}\right) X_{1}+1} \geq u_{p}\right\} \\
= & \begin{cases}1-\mathscr{P}\left\{X_{1} \leq \frac{k^{\prime}}{\left(a_{1} X_{1}+b_{1} Y_{1}\right)}\right\}, & \text { for }, u_{p}<\frac{\alpha}{(1-\alpha)}, \\
1-\mathscr{P}\left\{X_{1} \geq \frac{k^{\prime}}{\left(a_{1} X_{1}+b_{1} Y_{1}\right)}\right\}=0, & \text { otherwise. }\end{cases}
\end{aligned}
$$

where $u_{p}=2^{\left(2 R_{P U}\right)}-1, k^{\prime}=\frac{u_{p}}{a_{1}^{\prime}-u_{p} b_{1}^{\prime}}$. Now, the solution to (16) is obtained as (17) [23, Sec. 8.350.1, 8.352.1].

$$
\begin{gathered}
\mathscr{P}\left\{X_{1} \leq \frac{k^{\prime}}{\left(a_{1} X_{1}+b_{1} Y_{1}\right)}\right\}=\mathscr{P}\left(Y_{1} \leq \frac{k^{\prime}}{b_{1} X_{1}}-\frac{a_{1} X_{1}}{b_{1}}\right) \\
=\gamma\left(2,2 \sqrt{\frac{k^{\prime}}{a_{1}}}\right) \\
-\frac{4 \sum_{l=0}^{\infty} \sum_{t_{l}=0}^{l}\left(\begin{array}{l}
l \\
t_{l}
\end{array}\right)(-1)^{l}\left(\frac{k^{\prime}}{b_{1}}\right)^{l-t_{l}}\left(2-\frac{a_{1}}{b_{1}}\right)^{t_{l}}\left(\sqrt{\frac{k^{\prime}}{a_{1}}}\right)^{2 t_{l}-l+2}}{l !\left(2 t_{l}-l+2\right)} .
\end{gathered}
$$

Similarly, the probability of successful data transmission from $\mathrm{SU}_{1}$ to $\mathrm{PU}_{2}$ can be written as

$$
\begin{aligned}
& \mathscr{P}\left\{Y_{1} \leq \frac{k^{\prime \prime}}{\left(a_{1} X_{1}+b_{1} Y_{1}\right)}\right\}=\mathscr{P}\left(X_{1} \leq \frac{k^{\prime \prime}}{a_{1} Y_{1}}-\frac{b_{1} Y_{1}}{a_{1}}\right) \\
&= {\left[1-\exp \left(-\sqrt{\frac{k^{\prime \prime}}{b_{1}}}\right)\right]-\frac{\sum_{p_{a}=0}^{1} \sum_{r=0}^{p_{a}}(-1)^{r}\left(\begin{array}{c}
p_{a} \\
r
\end{array}\right)\left(\frac{b_{1}}{a_{1}}\right)^{r} \sum_{l=0}^{\infty} \sum_{t_{l}=0}^{l}\left(\begin{array}{l}
l \\
t_{l}
\end{array}\right)}{p_{a} !} } \\
& \frac{(-1)^{l} 2^{\left(l-t_{l}+p_{a}\right)}\left(\frac{k^{\prime \prime}}{a_{1}}\right)^{l+p_{a}-t_{l}-r}\left(1-\frac{2 b_{1}}{a_{1}}\right)^{t_{l}}\left(\sqrt{\frac{k^{\prime \prime}}{b_{1}}}\right)^{2 t_{l}-l+1+2 r-p_{a}}}{l !\left(2 t_{l}-l+1+2 r-p_{a}\right)} .
\end{aligned}
$$

where $k^{\prime \prime}=\frac{u_{p}}{a_{2}^{\prime}-u_{p} b_{2}^{\prime}}$.

The closed form solution to PU outage probability can be determined using (14), (17)-(18).

2) Outage Probability Analysis of Secondary System: SU outage probability ${ }^{4}$ using DF relaying, is shown as follows:

$$
\mathscr{P}_{\text {out }}^{(S U, D F)}=1-\overbrace{[\underbrace{\mathscr{P}\left\{Q_{1}\right\} \times \mathscr{P}\left\{Q_{2}\right\}}_{\text {MAC Phase }} \times \underbrace{\mathscr{P}\left\{R_{S U_{2}}^{(2, D F)} \geq R_{S U}\right\}}_{\text {BC Phase }}]}^{\text {Success Probability }}] .
$$

Similar to (13), the probability of success due to data transmission from $\mathrm{PU}_{i}(i \in 1,2)$ to $\mathrm{SU}_{2}$ is defined by the following three conditions together.

$Q_{2}=\left\{\begin{array}{l}R_{S U_{2}}^{(11)}=\frac{1}{2} \log _{2}\left(1+B_{1} X_{2}\right) \geq R_{P U_{1}}, \\ R_{S U_{2}}^{(12)}=\frac{1}{2} \log _{2}\left(1+B_{2} Y_{2}\right) \geq R_{P U_{2}}, \\ R_{S U_{2}}^{\sum_{2}}=\frac{1}{2} \log _{2}\left(1+B_{1} X_{2}+B_{2} Y_{2}\right) \geq R_{P U_{1}}+R_{P U_{2}} .\end{array}\right.$

where $B_{1}=\frac{P_{p_{1}}}{N_{a}\left(D_{4}\right)^{m} \sigma^{2}}, B_{2}=\frac{P_{p_{2}}}{N_{b}\left(D_{5}\right)^{m} \sigma^{2}}$.

$X_{2}=\sum_{n=1}^{N_{a}}\left|g_{1, n}\right|^{2}$ and $Y_{2}=\sum_{p=1}^{N_{b}}\left|g_{2, p}\right|^{2}$ follow the same nature of gamma distribution like $X_{1}$ and $\mathrm{Y}_{1}$, respectively.

${ }^{4}$ Since $\mathrm{SU}_{2}$ needs to decode the message of $\mathrm{SU}_{1}$ by removing PUs message in $\mathrm{BC}$ phase, therefore, successful information transmission from both PUs to $\mathrm{SU}_{1}$ is necessary in MAC phase. 
Similar to (14), based on (20), $\mathscr{P}\left\{Q_{2}\right\}$ can be expressed as follows

$$
\begin{aligned}
& \mathscr{P}\left\{Q_{2}\right\}=\left[\Gamma\left(2, \frac{2 u_{1}}{B_{1}}\right)-\left\{\gamma\left(2, \frac{2\left(u_{3}-u_{1}\right)}{B_{1}}\right)-\gamma\left(2, \frac{2 u_{1}}{B_{1}}\right)\right\}\right] \exp \left(-\frac{u_{2}}{B_{2}}\right) \\
& +\frac{4 \exp \left(-\frac{u_{3}}{B_{2}}\right)}{\left(2-\frac{B_{1}}{B_{2}}\right)^{2}}\left[\gamma\left\{2, \frac{\left(2-\frac{B_{1}}{B_{2}}\right)\left(u_{3}-u_{1}\right)}{B_{1}}\right\}-\gamma\left\{2, \frac{u_{1}\left(2-\frac{B_{1}}{B_{2}}\right)}{B_{1}}\right\}\right] .
\end{aligned}
$$

Following (11), the probability of successful data transmission between the link $\mathrm{SU}_{1}$ to $\mathrm{SU}_{2}$ is expressed as

$$
\mathscr{P}\left(R_{S U_{2}}^{(2, D F)} \geq R_{S U}\right)=\mathscr{P}\left(Z \geq \frac{u_{4}}{c\left(a_{1} X_{1}+b_{1} Y_{1}\right)}\right) .
$$

where $u_{4}=2^{\left(2 R_{S U}\right)}-1 . Z=\left|h_{3}\right|^{2}$ and it follows the gamma distribution with Nakagami shaping parameter $\mathrm{m}_{k}$. The PDF of $Z$ can be described as $f_{Z}(z)=\frac{z^{m_{k}-1} \exp \left(-\frac{z}{1 / m_{k}}\right)}{\Gamma\left(m_{k}\right)\left(1 / m_{k}\right)^{m_{k}}}$.

Now (22) can be evaluated as follows [23, Sec. 3.471.9, 8.352.2]:

Again for mathematical simplicity and tractability,

$$
\begin{gathered}
N_{a}=\mathbf{2}, N_{b}=\mathbf{1} \text { and } m_{k}=\mathbf{2} \text { are used } \\
\mathscr{P}\left(R_{S U_{2}}^{(2, D F)} \geq R_{S U}\right)=\frac{32}{\left(2-\frac{a_{1}}{b_{1}}\right)^{2}}\left[\left\{\frac{u_{4}}{2 c b_{1}}\right\} K_{2}\left\{2 \sqrt{\frac{2 u_{4}}{c b_{1}}}\right\}\right. \\
\left.-\frac{\sum_{j=0}^{1}\left\{\frac{u_{4}}{c a_{1}}\right\}^{\frac{(2-j)}{2}}\left\{\frac{u_{4}\left(2-\frac{a_{1}}{b_{1}}\right)}{c a_{1}}\right\}^{j}}{j !} K_{2-j}\left\{4 \sqrt{\frac{u_{4}}{c a_{1}}}\right\}\right] \\
+\left[\sum_{p_{a}=0}^{1} \frac{8(2)^{p_{a}}\left(\frac{u_{4}}{c a_{1}}\right)^{\frac{p_{a}+2}{2}} K_{2-p_{a}}\left(4 \sqrt{\frac{u_{4}}{c a_{1}}}\right)}{p_{a} !}\right] .
\end{gathered}
$$

Finally, the closed form SU outage expression using DF relaying can be determined using (14), (21) and (23).

\section{Rate And Outage Analysis of AF RElaying}

\section{A. Rate Analysis}

In the $\mathrm{BC}$ phase of $\mathrm{AF}$ relaying, $\mathrm{SU}_{1}$ broadcasts an amplified version of the signal generated after combining the PU signals, received in the MAC phase. To include this in the analysis, harvested power $\mathrm{P}_{s}$ in (5) is normalized using the factor $\xi$, expressed as 5

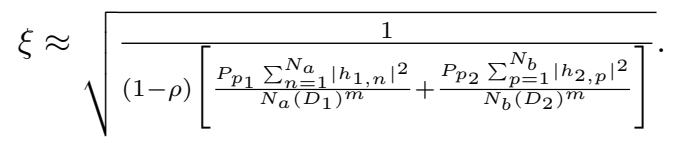

Based on the superposition coding and AF relaying principle $\mathrm{SU}_{1}$ uses $\alpha(0<\alpha<1)$ fraction of $P_{s}$ to relay the combined signal of PU information, the rest $(1-\alpha)$ portion is used to send its own independent message $X_{s}$ to $\mathrm{SU}_{2}$.

As both PUs know their individual transmitted signals, consequently, they are able to cancel their self-interference terms. Therefore, the instantaneous end-to-end SINR at $\mathrm{PU}_{i}$ can be expressed using (25) $\left(\mathrm{N}_{p}, \mathrm{~N}_{p}^{\prime} \in \mathrm{N}_{a}, \mathrm{~N}_{b} ; i, j \in 1,2\right.$; $i \neq \mathrm{j}$ ).

\footnotetext{
${ }^{5}$ As noise power is negligible with respect to the power received for information processing, therefore noise power is neglected.
}

$$
\begin{gathered}
\gamma_{i}^{A F}=\frac{\frac{\xi^{2} \alpha P_{s}(1-\rho) P_{p_{j}} \sum_{w=1}^{N_{p}} \sum_{w^{\prime}=1}^{N_{p}^{\prime}}\left|h_{i, w}\right|^{2}\left|h_{j, w^{\prime}}\right|^{2}}{N_{p}^{\prime}\left(D_{i} D_{j}\right)^{m}}}{\frac{(1-\alpha) P_{s} \sum_{w=1}^{N_{p}}\left|h_{i, w}\right|^{2}}{\left(D_{i}\right)^{m}}+\frac{\xi^{2} \alpha P_{s} \sum_{w=1}^{N_{p}}\left|h_{i, w}\right|^{2} \sigma^{2}}{\left(D_{i}\right)^{m}}+\sigma^{2}} \\
=\frac{C_{i} \sum_{w=1}^{N_{p}} \sum_{w=1}^{N_{p}^{\prime}}\left|h_{i, w}\right|^{2}\left|h_{j, w^{\prime}}\right|^{2}}{H_{i} \sum_{w=1}^{N_{p}}\left|h_{i, w}\right|^{4}+E_{i} \sum_{w=1}^{N_{p}} \sum_{w_{p}^{\prime}=1}^{N_{N_{p}^{\prime}}^{\prime}}\left|h_{i, w}\right|^{2}\left|h_{j, w^{\prime}}\right|^{2}+F_{i} \sum_{w=1}^{N_{p}}\left|h_{i, w}\right|^{2}+1} .
\end{gathered}
$$

where $\mathrm{C}_{1}=\frac{\eta \rho P_{p_{2}} \alpha}{N_{b} \sigma^{2}\left(D_{1}\right)^{m}\left(D_{2}\right)^{m}}, \mathrm{C}_{2}=\frac{\eta \rho P_{p_{1}} \alpha}{N_{a} \sigma^{2}\left(D_{1}\right)^{m}\left(D_{2}\right)^{m}}, \mathrm{H}_{1}$ $=\frac{(1-\alpha) \eta \rho P_{p_{1}}}{N_{a} \sigma^{2}\left(D_{1}\right)^{2 m}}, \mathrm{H}_{2}=\frac{(1-\alpha) \eta \rho P_{p_{2}}}{N_{b} \sigma^{2}\left(D_{2}\right)^{2 m}}, \mathrm{E}_{1}=\frac{(1-\alpha) \eta \rho P_{p_{2}}}{N_{b} \sigma^{2}\left(D_{1} D_{2}\right)^{m}}, \mathrm{E}_{2}=$ $\frac{(1-\alpha) \eta \rho P_{p_{1}}}{N_{a} \sigma^{2}\left(D_{1} D_{2}\right)^{m}}, \mathrm{~F}_{1}=\frac{\rho \alpha \eta}{(1-\rho)\left(D_{1}\right)^{m}}, \mathrm{~F}_{2}=\frac{\rho \alpha \eta}{(1-\rho)\left(D_{2}\right)^{m}}$.

Similarly, $\mathrm{SU}_{2}$ is able to detect PU signal by considering SU signal as an interference [24]. The instantaneous SINR can be expressed applying (26) (as shown at the top of the next page).

$$
\begin{aligned}
& \text { where } \quad \mathrm{U}_{1}=\frac{(1-\alpha) \eta \rho P_{p_{1}}}{\left(D_{3}\right)^{m} \sigma^{2} N_{a}\left(D_{1}\right)^{m}}, \quad \mathrm{~V}_{1}=\frac{(1-\alpha) \eta \rho P_{p_{2}}}{\left(D_{3}\right)^{m} \sigma^{2} N_{b}\left(D_{2}\right)^{m}}, \\
& \mathrm{U}_{2}=\frac{\alpha \eta \rho}{\left(D_{3}\right)^{m}(1-\rho)}, \quad \mathrm{U}_{3}=\frac{\alpha \eta \rho P_{p_{1}}}{\left(D_{3} D_{1}\right)^{m} \sigma^{2} N_{a}}, \quad \mathrm{~V}_{3}=\frac{\alpha \eta \rho P_{p_{2}}}{\left(D_{3} D_{2}\right)^{m} \sigma^{2} N_{b}} .
\end{aligned}
$$

The PU signals, being a strong one, $\mathrm{SU}_{2}$ first decodes $\mathrm{PU}$ signals considering $\mathrm{SU}_{1}$ signal as interference and removes it from the received signal. Then it decodes its own signal in the presence of noise. The instantaneous $\mathrm{SNR}$ at $\mathrm{SU}_{2}$ can be expressed in (27).

$$
\begin{array}{r}
\gamma_{s}^{A F}=\frac{\frac{(1-\alpha) \eta \rho\left|h_{3}\right|^{2}}{\left(D_{3}\right)^{m} \sigma^{2}}\left(\frac{P_{p_{1}} \sum_{n=1}^{N_{a}}\left|h_{1, n}\right|^{2}}{\left(D_{1}\right)^{m} N_{a}}+\frac{P_{p_{2}} \sum_{p=1}^{N_{b}}\left|h_{2, p}\right|^{2}}{\left(D_{2}\right)^{m} N_{b}}\right)}{\frac{\alpha \eta \rho\left|h_{3}\right|^{2}}{(1-\rho)\left(D_{3}\right)^{m}}+1} \\
=\frac{\left|h_{3}\right|^{2}\left(\sum_{n=1}^{N_{a}} U_{1}\left|h_{1, n}\right|^{2}+\sum_{p=1}^{N_{b}} V_{1}\left|h_{2, p}\right|^{2}\right)}{U_{2}\left|h_{3}\right|^{2}+1} .
\end{array}
$$

Achievable rate at $\mathrm{PU}_{i}$ is given by (based on the SINR)

$$
R_{P U_{i}}^{(2, A F)}=\frac{1}{2} \log _{2}\left\{1+\gamma_{i}^{A F}\right\} .
$$

The achievable rate for $\mathrm{PU}$ information decoding at $\mathrm{SU}_{2}$ is as

$$
R_{s, P U}^{(2, A F)}=\frac{1}{2} \log _{2}\left\{1+\gamma_{s, P U}^{A F}\right\} .
$$

The achievable rate at $\mathrm{SU}_{2}$ is given by (based on the $\mathrm{SNR}$ at $\mathrm{SU}_{2}$ )

$$
R_{S U_{2}}^{(2, A F)}=\frac{1}{2} \log _{2}\left\{1+\gamma_{s}^{A F}\right\}
$$

\section{B. Outage Probability Analysis}

1) Outage Probability of Primary System: PU outage probability, using AF relaying, is determined as follows:

$$
\mathscr{P}_{\text {out }}^{(P U, A F)}=1-\overbrace{\left[\mathscr{P}\left\{R_{P U_{1}}^{(2, A F)} \geq R_{P U_{1}}\right\} \times \mathscr{P}\left\{R_{P U_{2}}^{(2, A F)} \geq R_{P U_{2}}\right\}\right]}^{\text {Success Probability }} .
$$

Following (25), the probability of success due to data transmission between the link $\mathrm{SU}_{1}$ to $\mathrm{PU}_{1}$, is expressed as (32) $[23$, Sec. 3.471.9, 8.352.1] (as shown at the top of the next page).

The symbol $\mathrm{K}_{v}($.$) indicates the modified Bessel function.$ Similarly, it is also possible to determine the probability of successful data transmission between the link $\mathrm{SU}_{1}$ to $\mathrm{PU}_{2}$. 


$$
\gamma_{s, P U}^{A F}=\frac{\frac{\alpha \eta \rho\left|h_{3}\right|^{2}}{\left(D_{3}\right)^{m} \sigma^{2}}\left(\frac{P_{p_{1}} \sum_{n=1}^{N_{a}\left|h_{1, n}\right|^{2}}}{\left(D_{1}\right)^{m} N_{a}}+\frac{P_{p_{2}} \sum_{p=1}^{N_{b}}\left|h_{2, p}\right|^{2}}{\left(D_{2}\right)^{m} N_{b}}\right)}{\frac{(1-\alpha) \eta \rho\left|h_{3}\right|^{2}}{\left(D_{3}\right)^{m} \sigma^{2}}\left(\frac{P_{p_{1}} \sum_{n=1}^{N_{a}\left|h_{1, n}\right|^{2}}}{\left(D_{1}\right)^{m} N_{a}}+\frac{P_{p_{2}} \sum_{p=1}^{N_{b}}\left|h_{2, p}\right|^{2}}{\left(D_{2}\right)^{m} N_{b}}\right)+\frac{\alpha \eta \rho\left|h_{3}\right|^{2}}{(1-\rho)\left(D_{3}\right)^{m}}+1}=\frac{\left|h_{3}\right|^{2}\left(\sum_{n=1}^{N_{a}} U_{3}\left|h_{1, n}\right|^{2}+\sum_{p=1}^{N_{b}} V_{3}\left|h_{2, p}\right|^{2}\right)}{\left|h_{3}\right|^{2}\left(\sum_{n=1}^{N_{a}} U_{1}\left|h_{1, n}\right|^{2}+\sum_{p=1}^{N_{b}} V_{1}\left|h_{2, p}\right|^{2}\right)+U_{2}\left|h_{3}\right|^{2}+1} .
$$

$$
\begin{aligned}
\mathscr{P}\left(R_{P U_{1}}^{(2, A F)}\right. & \left.\geq R_{P U_{1}}\right)=\mathscr{P}\left[\frac{C_{1} \sum_{n=1}^{N_{a}}\left|h_{1, n}\right|^{2} \sum_{p=1}^{N_{b}}\left|h_{2, p}\right|^{2}}{H_{1} \sum_{n=1}^{N_{a}}\left|h_{1, n}\right|^{4}+E_{1} \sum_{n=1}^{N_{a}}\left|h_{1, n}\right|^{2} \sum_{p=1}^{N_{b}}\left|h_{2, p}\right|^{2}+F_{1} \sum_{n=1}^{N_{a}}\left|h_{1, n}\right|^{2}+1} \geq u_{1}\right] \\
& =\mathscr{P}\left[\frac{C_{1} X_{1} Y_{1}}{H_{1} X_{1}{ }^{2}+E_{1} X_{1} Y_{1}+F_{1} X_{1}+1} \geq u_{1}\right]=1-\mathscr{P}\left[Y_{1}<\frac{F_{1} u_{1}}{\left(C_{1}-E_{1} u_{1}\right)}+\frac{H_{1} u_{1} X_{1}}{\left(C_{1}-E_{1} u_{1}\right)}+\frac{u_{1}}{\left(C_{1}-E_{1} u_{1}\right) X_{1}}\right]
\end{aligned}
$$

Putting, the values of $N_{a}=2$ and $N_{b}=1$;

$$
\left.=8\left\{\exp \left(-\frac{F_{1} u_{1}}{\left(C_{1}-E_{1} u_{1}\right)}\right)\right\}\left(\frac{u_{1} /\left(C_{1}-E_{1} u_{1}\right)}{2+\frac{H_{1} u_{1}}{\left(C_{1}-E_{1} u_{1}\right)}}\right)\right\} K_{2}\left\{2 \sqrt{\frac{u_{1}}{\left(C_{1}-E_{1} u_{1}\right)}\left(2+\frac{H_{1} u_{1}}{C_{1}-E_{1} u_{1}}\right)}\right\} \text {. }
$$

2) Outage Probability Analysis of Secondary System: SU outage probability, using AF relaying mechanism, can be determined as follows:

$$
\begin{aligned}
& \mathscr{P}_{\text {out }}^{(S U, A F)}=1-[\underbrace{\mathscr{P}\left\{R_{S U_{2}}^{(2, A F)} \geq R_{S U} \mid R_{s, P U}^{(2, A F)} \geq R_{P U}\right\}}_{\text {Success of } \mathbf{S U}_{1} \rightarrow \mathbf{S U}_{2}} \\
& \times \underbrace{\mathscr{P}\left\{R_{s,}^{(2, A F)} \geq R_{P U}\right\}}_{\text {Successful decoding of PU signals at }}] \text {. }
\end{aligned}
$$

Following (27), the probability of successful data transmission between the link $\mathrm{SU}_{1}$ to $\mathrm{SU}_{2}$ is expressed using (34) [23, Sec. 3.471.9, 8.352.2].

$$
\mathscr{P}\left(R_{S U_{2}}^{(2, A F)} \geq R_{S U}\right)=\mathscr{P}\left\{\frac{\left|h_{3}\right|^{2}\left(U_{1} \sum_{n=1}^{N_{a}}\left|h_{1, n}\right|^{2}+V_{1} \sum_{p=1}^{N_{b}}\left|h_{2, p}\right|^{2}\right.}{U_{2}\left|h_{3}\right|^{2}+1} \geq u_{4}\right\}
$$

For mathematical simplicity and tractability, the values are set as,

$$
\begin{aligned}
& N_{a}=\mathbf{2}, N_{b}=1 \text { and } m_{k}=\mathbf{2} \\
& =\frac{32 \exp \left(-\frac{u_{4} U_{2}}{V_{1}}\right)}{\left(2-\frac{U_{1}}{V_{1}}\right)^{2}}\left[\left(\frac{u_{4} K_{2}\left(2 \sqrt{\frac{2 u_{4}}{V_{1}}}\right)}{2 V_{1}}\right)-\frac{\sum_{j=0}^{1} \exp \left\{-\left(\frac{u_{4} U_{2}}{U_{1}}\right)\left(2-\frac{U_{1}}{V_{1}}\right)\right\}}{j !}\right. \\
& \left.\left(2-\frac{U_{1}}{V_{1}}\right)^{j} \sum_{t_{l}=0}^{j}\left(\begin{array}{c}
j \\
t_{l}
\end{array}\right)\left(\frac{u_{4} U_{2}}{U_{1}}\right)^{j-t_{l}}\left(\frac{u_{4}}{U_{1}}\right)^{\frac{2+t_{l}}{2}} K_{2-t_{l}}\left(4 \sqrt{\frac{u_{4}}{U_{1}}}\right)\right]+[8 \\
& \left.\frac{\sum_{p_{a}=0}^{1} \sum_{r=0}^{p_{a}}\left(\begin{array}{c}
p_{a} \\
r
\end{array}\right)(2)^{p_{a}}\left(\frac{u_{4}}{U_{1}}\right)^{\frac{2 p_{a}+2-r}{2}}\left(U_{2}\right)^{p_{a}-r} \exp \left(-\frac{2 u_{4} U_{2}}{U_{1}}\right) K_{2-r}\left(4 \sqrt{\frac{u_{4}}{U_{1}}}\right)}{p_{a} !}\right] .
\end{aligned}
$$

Similar to (34), the probability of success for the decoding of the PU information in presence of SU interference and noise can be expressed using (35) (as shown at the top of the next page).

$$
\begin{aligned}
& \text { where } S_{1}=\frac{\eta \rho P_{p_{1}}}{\left(D_{3}\right)^{m} \sigma^{2} N_{a}\left(D_{1}\right)^{m}}, S_{2}=\frac{\eta \rho P_{p_{2}}}{\left(D_{3}\right)^{m} \sigma^{2} N_{a}\left(D_{2}\right)^{m}}, u_{s}= \\
& \frac{u_{p}}{\alpha-u_{p}(1-\alpha)} \text {. }
\end{aligned}
$$

Finally, the closed form SU outage expression using AF relaying can be determined using (34) and (35). One may find the detail derivation in [25].

One of the main objective of this paper is to explore the effect of multiple antennas on the outage performance of the two-way PU and one-way SU communication. The use of multiple antennas makes the mathematical analysis quite complex, involving several summations in the closed form expressions of PU and SU outage probability. It may be simplified further by considering $N_{a}=N_{b}=1$, which represents the mathematical analysis in a compact form. Simulation results are shown in the next section to support the mathematical analysis.

\section{Numerical RESUlTS AND Discussions}

Numerical values for the system parameters used are enlisted in Table-2. The constraint of the maximum PU outage limit (say 0.1) is found to be met at $\alpha=0.81$ by both AF and DF relaying for $\mathrm{N}_{a}=2$. This value of $\alpha$ is used for the results shown in Fig. 4, Fig. 5 and Fig. 6. Considering the practicability of the use of the number of transmitting antennas on mobile devices in present technology, we set $\mathrm{N}_{b}=1$ in our simulation results.

TABLE II

SET OF NUMERICAL VALUES OF NECESSARY PARAMETERS

\begin{tabular}{l|l}
\hline \hline Name & Value \\
\hline \hline$R_{P U_{1}}=R_{P U_{2}}$ & $\begin{array}{l}0.2 \mathrm{bps} / \mathrm{Hz} \text { (Fig. 3, Fig. 4, Fig. } \\
6 \text { ) }\end{array}$ \\
\hline$R_{P U_{1}}, R_{P U_{2}}$ & $0.5 \mathrm{bps} / \mathrm{Hz}, 0.2 \mathrm{bps} / \mathrm{Hz}$ (Fig. 4, \\
& Fig. 5) \\
\hline$R_{S U}$ & $1 \mathrm{bps} / \mathrm{Hz}$ \\
\hline $\mathrm{D}_{\text {sistance between } \mathrm{PU}_{1} \text { and } \mathrm{PU}_{2} \text { (L) }}$ & $20 \mathrm{~m}[18]$ \\
\hline $\mathrm{D}_{1}=\mathrm{D}_{2}=\mathrm{D}_{4}=\mathrm{D}_{5}$ & $10 \mathrm{~m}$ \\
\hline Distance between $\mathrm{SU}_{1}$ and $\mathrm{SU}_{2}\left(\mathrm{D}_{3}\right)$ & $10 \mathrm{~m}$ \\
\hline PU transmit power $\left(P_{p_{1}}=P_{p_{2}}=P_{p}\right)$ & $-23 \mathrm{dBm}$ (Fig. 3 to Fig. 5), -50 \\
& dBm to -15 dBm (Fig. 6) \\
\hline Average noise power $\left(n_{p u}=n_{s u}=n_{p}\right)$ & $-100 \mathrm{dBm}[11]$ \\
\hline Path loss exponent $(m)$ & 2.7 \\
\hline Energy conversion efficiency $(\eta)$ & 0.9 \\
\hline Power splitting factor $(\rho)$ & 0.9 \\
\hline \hline
\end{tabular}




$$
\begin{aligned}
& \mathscr{P}\left\{R_{s, P U}^{(2, A F)} \geq R_{P U}\right\}=\mathscr{P}\left\{\frac{Z\left(U_{3} X_{1}+V_{3} Y_{1}\right)}{Z\left(U_{1} X_{1}+V_{1} Y_{1}\right)+U_{2} Z+1} \geq u_{p}\right\}= \begin{cases}\mathscr{P}\left\{Z\left(S_{1} X_{1}+S_{2} Y_{1}\right) \geq u_{s} U_{2} Z+u_{s}\right\}, & \text { for }, u_{s}<\frac{\alpha}{(1-\alpha)}, \\
0, & \text { otherwise. }\end{cases} \\
& = \begin{cases}\frac{32 \exp \left(-\frac{u_{s} U_{2}}{S_{2}}\right)}{\left(2-\frac{S_{1}}{S_{2}}\right)^{2}}\left[\left(\frac{u_{s} K_{2}\left(2 \sqrt{\frac{2 u_{s}}{S_{2}}}\right)}{2 S_{2}}\right)-\frac{\left.\sum_{j=0}^{1} \exp \left\{-\left(\frac{u_{s} U_{2}}{S_{1}}\right)\left(2-\frac{S_{1}}{S_{2}}\right)\right\}\left(2-\frac{S_{1}}{S_{2}}\right)^{j} \sum_{t_{l}=0}^{j}\left(\begin{array}{l}
j \\
t_{l}
\end{array}\right)\left(\frac{u_{s} U_{2}}{S_{1}}\right)^{j-t_{l}}\right]\left(\frac{u_{s}}{S_{1}}\right)^{\frac{2+t_{l}}{2}} K_{2-t_{l}}\left(4 \sqrt{\frac{u_{s}}{S_{1}}}\right)}{j !}\right] ; & \text { for, } u_{s}<\frac{\alpha}{(1-\alpha)} ; \\
+\left[\sum_{p_{a}=0}^{1} \frac{8 \sum_{r=0}^{p_{a}}\left(\begin{array}{c}
p_{a} \\
r
\end{array}\right)(2)^{p_{a}}\left(\frac{u_{s}}{S_{1}}\right)^{\frac{2 p_{a}+2-r}{2}}\left(U_{2}\right)^{p_{a}-r} \exp \left(-\frac{2 u_{s} U_{2}}{S_{1}}\right) K_{2-r}\left(4 \sqrt{\frac{u_{s}}{S_{1}}}\right)}{p_{a} !} \quad\right. \text { otherwise. } & \\
0 ; & \end{cases}
\end{aligned}
$$

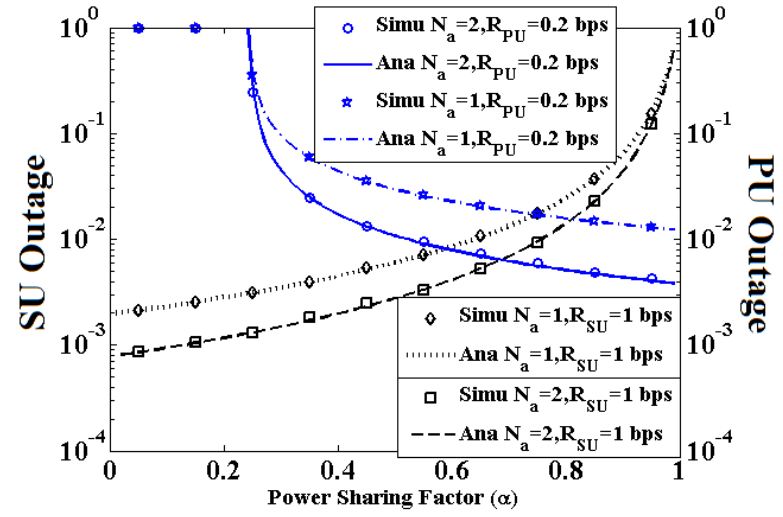

(a)

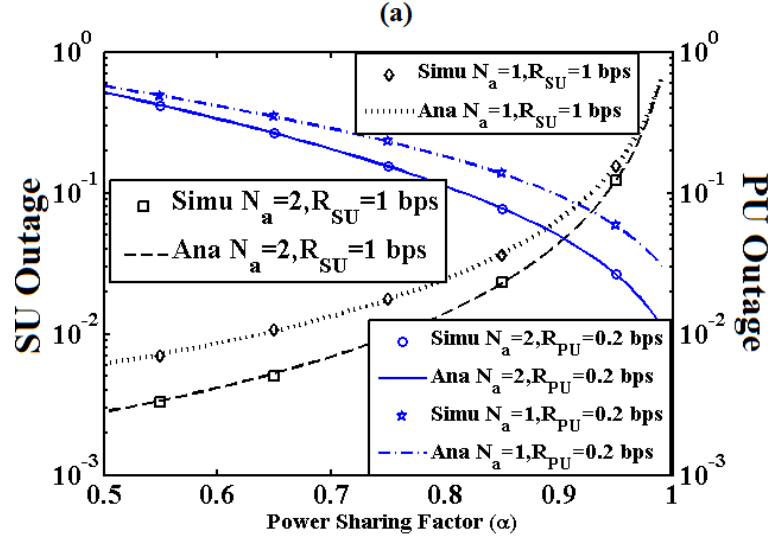

(b)

Fig. 3. Outage Probability vs. $\alpha$ : (a) DF relaying (b) AF relaying

The outage performances of both PU and SU systems are shown as variation in power-sharing factor $(\alpha)$ for both $\mathrm{DF}$ and AF relaying in Fig. 3.a and Fig. 3.b. It is clearly seen that the analytical results match perfectly with the simulation results. For the small value of $\alpha$, the higher fraction of the harvesting power is allocated to SU information transmission and small fraction of power is used for DF relaying of PU information. Thus SU outage is found to be low while PU outage to be high. The reverse situation is to observed at high value of $\alpha$. The small value of $\alpha$ (close to zero (0)) causes $\mathrm{PU}$ outage of AF relaying same to that of DF relaying and SU performance is found to be the worst by following (33)-
(35). Since $50 \%$ or more power is assigned to PU information transmission to decode PU signals perfectly in presence of SU interference, therefore the minimum value of $\alpha$ is shown as 0.5 for AF relaying in Fig. 3.b. The threshold limit of $10 \%$ PU outage is achieved at $\alpha=0.29$ and $\mathrm{N}_{a}=1$ for DF relaying and the same outage limit is achieved at $\alpha=0.89$ and $\mathrm{N}_{a}=1$ for $\mathrm{AF}$ relaying. If the number of antennas is increased then PU performance is improved accordingly. If $\mathrm{N}_{a}$ is increased from 1 to 2 , about $46 \%$ improvement (reduction) in PU outage is observed for DF relaying and about $39 \%$ improvement of PU outage is observed for AF relaying. If $\mathrm{N}_{a}$ is increased from 1 to 2, about $58 \%$ improvement of SU outage is observed at $\alpha=0.27$ and $\mathrm{R}_{S U}=1$ bps for DF relaying and about $42 \%$ improvement in SU outage is observed at $\alpha=0.81$ and $\mathbf{R}_{S U}=1$ bps for AF relaying.

Fig. 4 and 5 show the outage performances of PU and $\mathrm{SU}$, respectively with respect to power splitting factor $(\rho)$ for both DF and AF relaying mechanisms. As depicted in the figures, the outage performances of both $\mathrm{PU}$ and $\mathrm{SU}$ are very poor for $\rho \rightarrow 0$ and $\rho \rightarrow 1$. Initially, the performance of PU and SU outage are improved with the increase in the value of $\rho$, and they attain their minimum values of outage at the optimal value of $\rho^{*}$. Thereafter, when the value of $\rho$ is increased further, it leads to an increase in both PU and SU outage. The reason behind the characteristics of this graphical plot can be explained as follows. Initially, when $\rho$ value is very low, the energy harvested at $\mathrm{SU}_{1}$ is insufficient to broadcast the information at the $\mathrm{BC}$ phase and effectively the outage performance of both $\mathrm{PU}$ and SU are very poor. Now if the value of $\rho$ increases, the harvesting energy at $\mathrm{SU}_{1}$ is also increased and consequently both $\mathrm{PU}$ and SU outage performance is improved accordingly. Further degradation on the outage performance is found due to major power allocation for $\mathrm{EH}$ and less power allocation for decoding the information received from PU to SU signal transmission. Performance of PU outage is significantly improved for DF relaying as compared to $\mathrm{AF}$ relaying mechanism whereas the SU outage performance looks almost same for both the relaying mechanisms with respect to $\rho$. The overall outage performances for both PU and SU are improved with the increase in the number of antennas used at PU nodes. As shown in Fig. 4., the performance of PU outage is deteriorating 


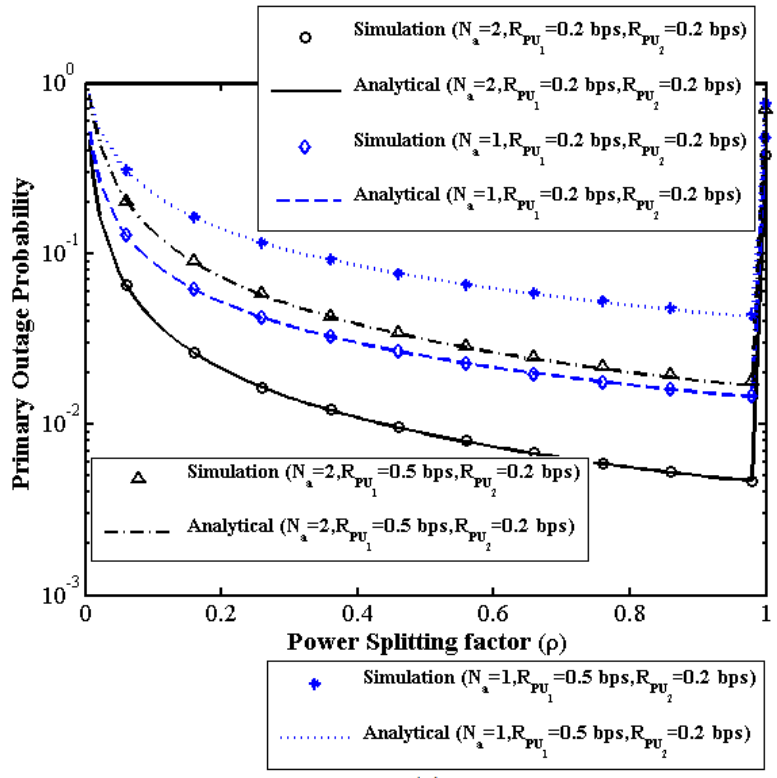

(a)

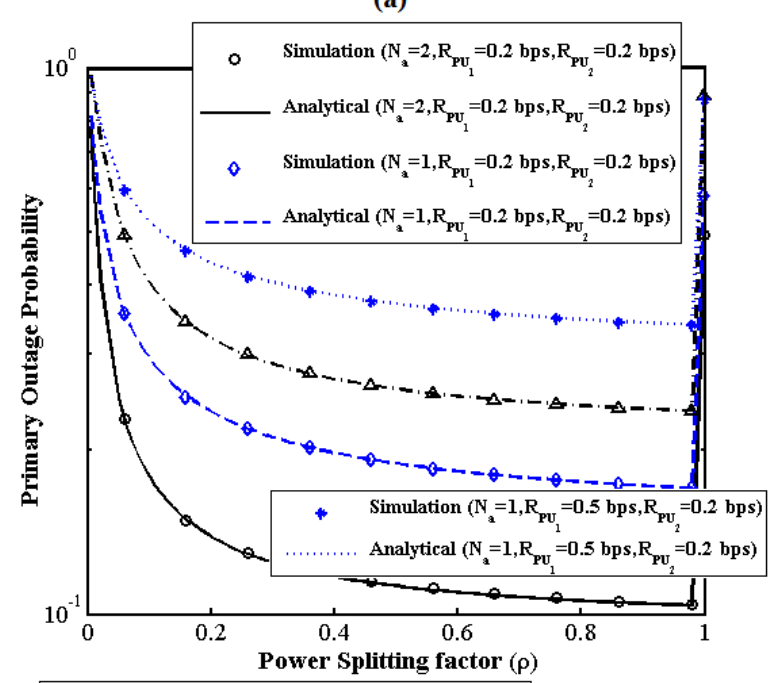

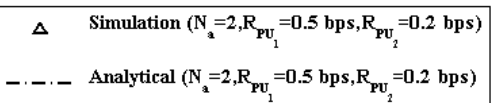

(b)

Fig. 4. PU Outage Probability vs. $\rho$ : (a) DF relaying, (b) AF relaying

with the increasing downlink data rate. In case of SU outage probability, the performance seems almost same for equal data rate in uplink-downlink transmission and different data rates in uplink-downlink transmission. Thus, SU outage performance is shown in Fig. 5 for the different data rates in uplinkdownlink transmission.

Now SE and EE of the system can be defined as [13]

$$
\eta_{S E}=\frac{2 \times\left(1-\mathscr{P}_{\text {out }}^{P U}\right) \times R_{P U} \times T}{2 T}+\frac{\left(1-\mathscr{P}_{\text {out }}^{S U}\right) \times R_{S U} \times T}{2 T} .
$$

EE can be defined as the ratio of SE to the total energy consumed for PU transmission [26].

The SE and EE performances of the DF and AF relaying protocols are shown with respect to the PU transmit power

\footnotetext{
${ }^{6}$ The transmit power is considered to be normalized with respect to average channel noise power.
}

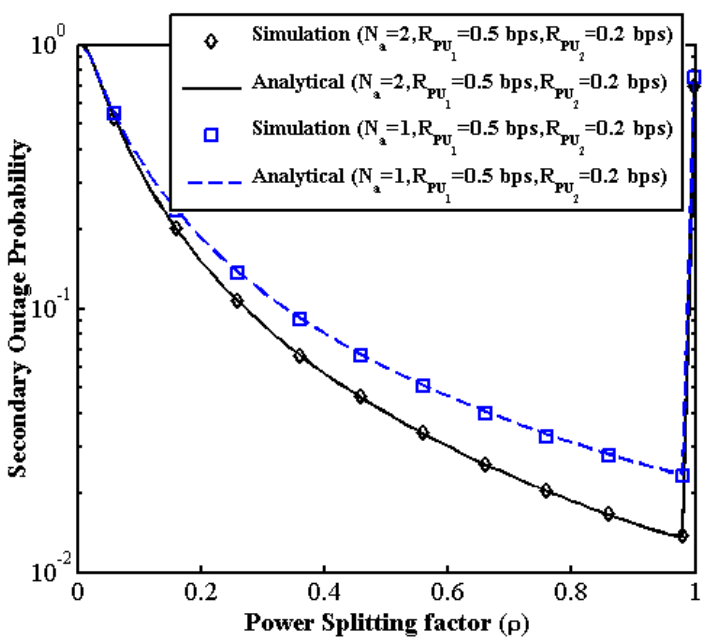

(a)

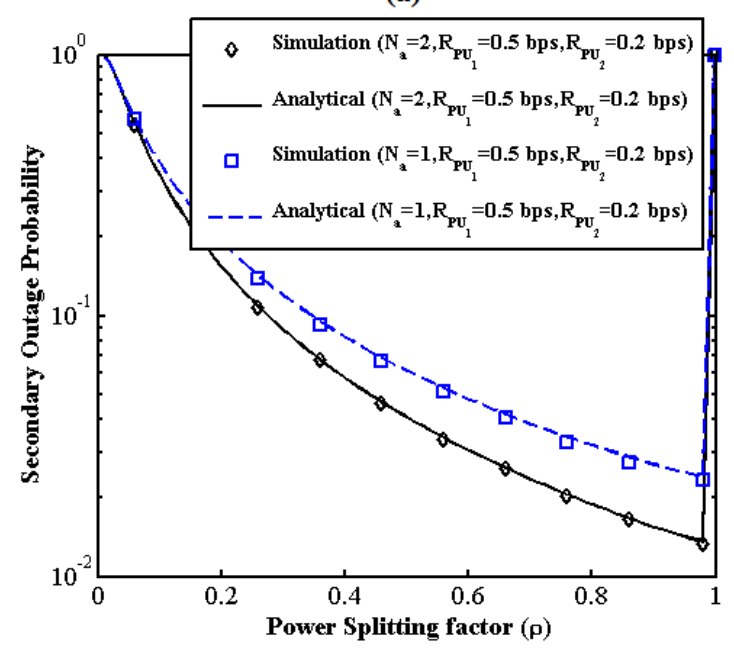

(b)

Fig. 5. SU Outage Probability vs. $\rho$ : (a) DF relaying, (b) AF relaying

in Fig. 6a and Fig. 6b, respectively. The performance of the proposed system using $\mathrm{AF}$ and $\mathrm{DF}$ relaying schemes are compared with the similar system supporting two-way PU and one-way SU communication simultaneously [13]. As shown in figure, $\mathrm{SE}$ is improved and EE is deteriorated with the increase in PU transmit power. The characteristics of this graphical plot may be explained as follows. As transmission power of PUs are increased, the PU and SU outage probabilities are decreased accordingly following (14)-(18), (21), (23), (32), (34) and (35) for DF and AF relaying mechanisms, respectively. As inverse relationship is maintained between SE and the outage probability of both PU and SU system, the $\mathrm{SE}$ is improved with the increase in the transmission power of PU. Less increment of SE as compared to more energy consumption leads to a degradation of $\mathrm{EE}$ with the increasing value of PUs' transmit power.

Mathematical analysis and consequent simulation results show that our proposed system using DF relaying mechanism is more efficient as compared to AF relaying mechanism and similar model of two-way PU and one-way SU communications [13]. In term of SE, our proposed system using DF relaying mechanism shows $224 \%, 196 \%$ and $142 \%$ improved 


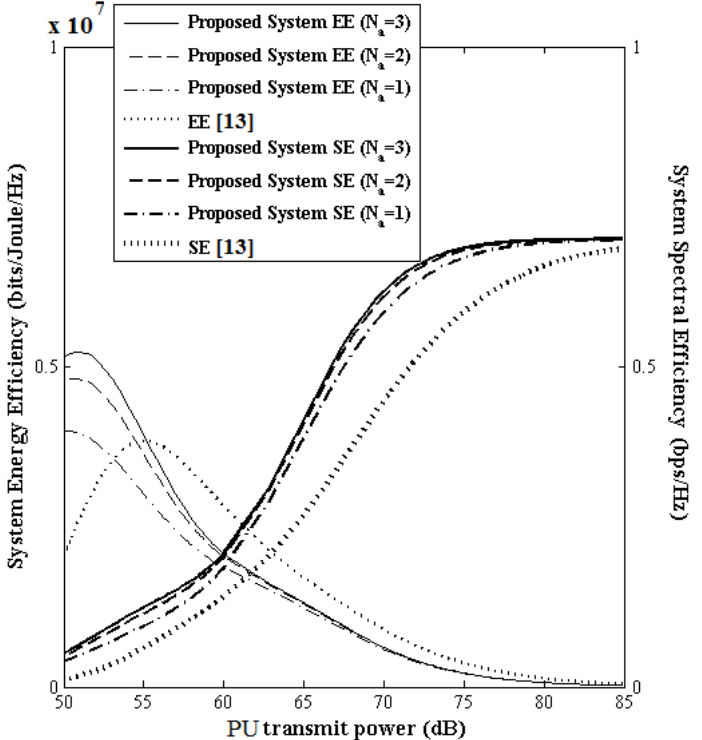

(a)

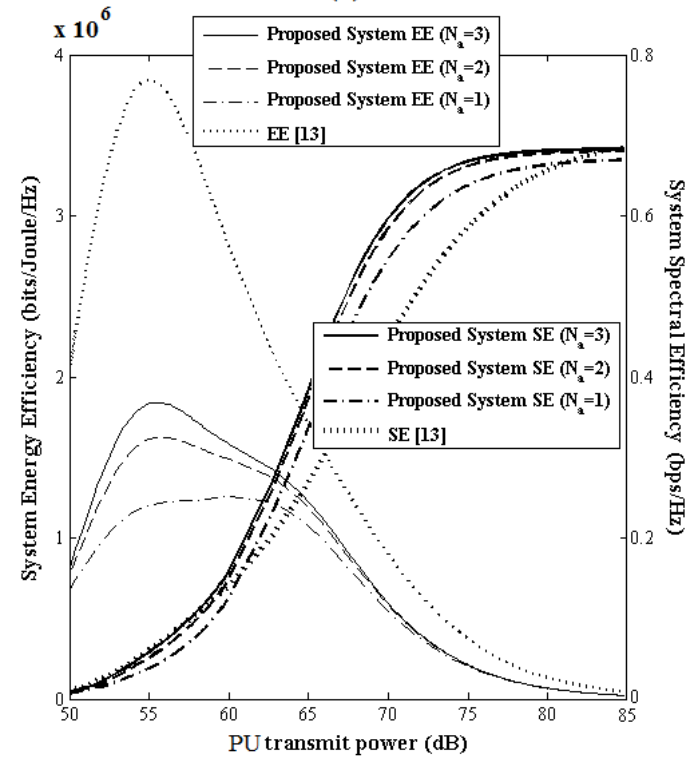

(b)

Fig. 6. System SE and EE vs. total PU transmit power : (a) DF relaying (b) $\mathrm{AF}$ relaying

performance compared to [13] for $\mathrm{N}_{a}=3, \mathrm{~N}_{a}=2$ and $\mathrm{N}_{a}=1$, respectively at $52 \mathrm{~dB}$ transmit power. Our system using $\mathrm{AF}$ relaying shows performance $11 \%, 21 \%$ and $38 \%$ inferior to [13] in term of SE for the given values of $\mathrm{N}_{a}=3, \mathrm{~N}_{a}=2$ and $\mathrm{N}_{a}=1$, respectively at the same value of $\mathrm{PU}$ transmit power. However, it is also noted from Fig. $6 \mathrm{~b}$ that AF relaying shows gradual improvement with the increase in PU transmit power and becomes comparable to the achievable improvement of DF relaying (Fig. 6a). It is also observed that the improvement of $\mathrm{SE}$ is more as antenna number increases from 1 to 2 over the increase in 2 to 3 . In term of peak EE, our proposed system using DF relaying mechanism is shown $62 \%, 48 \%$ and $21 \%$ improved performance compared to [13] and while performance of [13] is $56 \%, 60 \%$ and $69 \%$ better compared to the proposed system using $\mathrm{AF}$ relaying for $\mathrm{N}_{a}=3, \mathrm{~N}_{a}=2$ and $\mathrm{N}_{a}=1$, respectively at $52 \mathrm{~dB}$ transmit power. However, the proposed model is less energy efficient at high transmit power compared to [13].

\section{CONCLUSIONS}

This paper has investigated the scope of SWIPT enabled IoT communication, on the licensed spectrum using overlay spectrum sharing mode of cognitive radio networks. Transmitting IoD node harvests energy from the information bearing RF signals of both eNB and UE transmission and this energy is used in relaying between eNB and UE. Apart from the close match between the analytical and simulation results on the outage experiences in IoD and cellular systems, it is also found that DF relaying mechanism is more efficient over $\mathrm{AF}$ relaying in terms of $\mathrm{EE}$ and $\mathrm{AF}$ relaying is more sensitive to the impact of the number of antennas used by the cellular system. The proposed network architecture and the spectrum sharing model can be extended further (i) to analyse the similar network performance by considering the impacts of both power splitting and time switching factors together, (ii) to analyse the outage for more realistic non-linear RFEH model, (iii) to improve the secrecy of the relay assisted cellular communication using the PUs' signals for friendly jamming in addition to EH and (iv) to study a game theoretic modelling of the possible negotiations between the cellular users and multiple IoT device pairs for an efficient trading of the available radio resources of the former.

\section{REFERENCES}

[1] X. Liu and N. Ansari, "Green Relay Assisted D2D Communications With Dual Batteries in Heterogeneous Cellular Networks for IoT,' IEEE Internet of Things Journal, vol. 4, no. 5, pp. 1707 - 1715, Oct. 2017.

[2] L. Chettri and R. Bera, "A Comprehensive Survey on Internet of Things (IoT) Toward 5G Wireless Systems," IEEE Internet of Things Journal, vol. 7, no. 1, pp. 16 - 32, Jan. 2020.

[3] L. Zhang, Y. -C. Liang, and M. Xiao , "Spectrum Sharing for Internet of Things: A Survey," IEEE Wireless Communications Journal, vol. 26, no. 3, pp. 132-139, Jun. 2019.

[4] D. S. Gurjar and P. K. Upadhyay, "Overlay Device-to-Device Communications in Asymmetric Two-Way Cellular Systems With Hybrid Relaying," IEEE Systems Journal, vol. 12, no. 4, pp. 3713-3724, Dec. 2018.

[5] A. Hyadi, E. M. Driouch, W. Ajib, and M.-S. Alouini, "Overlay Cognitive Radio Systems with Adaptive Two-Way Relaying," In Proceedings of IEEE Global Communications Conference (GLOBECOM), pp. 937-942, Atlanta, GA, USA, Dec. 2013.

[6] Y. Han, A. Pandharipande, and S. H. Ting, "Cooperative Decode-andForward Relaying for Secondary Spectrum Access," IEEE Transactions on Wireless Communications, vol. 8, no. 10, pp. 4945-4950, Oct. 2009.

[7] H. Ju, and R. Zhang, "Throughput maximization in wireless powered communication networks," IEEE Transactions on Wireless Communications, vol. 13, no. 1, pp. 418 - 428, Jan. 2014.

[8] X. Zhou, R. Zhang, and C. K. Ho, "Wireless Information and Power Transfer: architecture design and rate-energy tradeoff," IEEE Transactions on communications, vol. 61, no. 11, pp. 4754 - 4767, Nov. 2013.

[9] W. Sun, Q. Song, J. Zhao, L. Guo and A. Jamalipour, "Adaptive Resource Allocation in SWIPT-Enabled Cognitive IoT Networks," IEEE Internet of Things Journal, DOI: 10.1109/JIOT.2021.3084472, pp. 1 - 11, May. 2021.

[10] A. A. Nasir, X. Zhou, S. Durrani, and R. A. Kennedy, "Relaying Protocols for Wireless Energy Harvesting and Information Processing," IEEE Transactions on Wireless Communications, vol. 12, no. 7, pp. 36223636, Jul. 2013.

[11] N. Jain, and V. Bohara, "Energy Harvesting and Spectrum Sharing Protocol for Wireless Sensor Networks," IEEE Wireless Communications Letters, vol. 4, no. 6, pp. 697-700, Dec. 2015. 
[12] Z. Wang, Z. Chen, Y. Yao, B. Xia, and H. Liu, "Wireless Energy Harvesting and Information Transfer in Cognitive Two-Way Relay Networks," In Proceedings of IEEE Global Communications Conference (GLOBECOM), pp. 3465-3470, Austin, Tx, Dec. 2014.

[13] A. Mukherjee, T. Acharya, and M. R. A. Khandaker, "Outage Analysis for SWIPT-Enabled Two-Way Cognitive Cooperative Communications," IEEE Transactions on Vehicular Technology, vol. 67, no. 9, pp. 90329036, Sep. 2018.

[14] S. Ghosh, T. Acharya, and S. P. Maity, "On Outage Analysis in SWIPT Enabled Bidirectional D2D Communications using Spectrum Sharing in Cellular Networks," IEEE Transactions on Vehicular Technology, vol. 69, no. 9, pp. 10167 - 10176, Sep. 2020.

[15] T. Kalluri, M. Peer, V. Ashok Bohara, D. B. da Costa and U. S. Dias, "Cooperative spectrum sharing-based relaying protocols with wireless energy harvesting cognitive user," IET Communications, vol. 12, no. 7, pp. 838-847, May 2018.

[16] S. Ghosh, T. Acharya, and S. P. Maity, "On Outage Analysis in Cooperative Cognitive Radio Network with RF Energy Harvesting," In Proceedings of IEEE International Conference on Signal Processing and Communications (SPCOM), pp. 1-5, Bangalore, India, Jul. 2018.

[17] S. Suyama, T. Okuyama, Y. Inoue, and Y. Kishiyama, "5G multi-antenna technology," NTT Docomo Technical Journal, vol. 17, no. 4, pp. 29-39, Apr. 2016.

[18] V. Chandrasekhar, J. G. Andrews, and A. Gatherer, "Femtocell networks: a survey," IEEE Communications Magazine, vol. 46, no. 9, pp. 59-67, Sep. 2008.

[19] S. M. Alamouti, "A simple transmit diversity technique for wireless communications," IEEE Journal on Selected Areas in Communications, vol. 16, no. 8, pp. 1451-1458, Oct. 1998.

[20] G. T. Freitas de Abreu, "GABBA Codes: Generalized full-rate orthogonally decodable space-time block codes," In Proceedings of Thirty-Ninth Asilomar Conference on Signals, Systems and Computers, pp. 1278-1283, Pacific Grove, CA, USA, Oct. 2005.

[21] V. Tarokh, N. Seshadri and A. R. Calderbank, "Space-time codes for high data rate wireless communication: performance criterion and code construction," IEEE Transactions on Information Theory, vol. 44, no. 2, pp. 744-765, Mar. 1998

[22] D. Tse, and P. Viswanath, "Fundamentals of wireless communication," Cambridge University press, 2005.

[23] I. S. Gradshteyn, and I. M. Ryzhik, "Table of Integrals, Series, and Products," Academic Press, Edition 7, 2007.

[24] Y. Saito, Y. Kishiyama, A. Benjebbour, T. Nakamura, A. Li and K. Higuchi, "Non-Orthogonal Multiple Access (NOMA) for Cellular Future Radio Access," In Proceedings of IEEE 77th Vehicular Technology Conference (VTC Spring), pp. 1-5, Dresden, Germany, Jun. 2013.

[25] S. Ghosh, T. Acharya, and S. P. Maity, "Outage Analysis in SWIPT Enabled Cooperative AF/DF Relay Assisted Two-Way Spectrum Sharing Communication," available at https://arxiv.org/pdf/2007.08599.pdf, 2020.

[26] Y. Liu, L. Wang, M. Elkashlan, T. Q. Duong, and A. Nallanathan, "Twoway relay networks with wireless power transfer: Design and performance analysis," IET Communications, vol. 10, no. 14, pp. 1810-1819, Jun. 2016. 